Sādhanā Vol. 36, Part 3, June 2011, pp. 281-292. (c) Indian Academy of Sciences

\title{
Identification of bilinear systems using differential evolution algorithm
}

\author{
SABAN OZER and HASAN ZORLU* \\ Department of Electirical and Electronic Engineering, Erciyes University, \\ 38039 Kayseri, Turkey \\ e-mail: hzorlu@erciyes.edu.tr
}

MS received 26 August 2010; revised 24 December 2010; accepted 18 February 2011

\begin{abstract}
In this work, a novel identification method based on differential evolution algorithm has been applied to bilinear systems and its performance has been compared to that of genetic algorithm. Box-Jenkins system and different type bilinear systems have been identified using differential evolution and genetic algorithms. The simulation results have shown that bilinear systems can be successfully and efficiently identified using these algorithms.
\end{abstract}

Keywords. System identification; bilinear system; differential evolution algorithm; box-Jenkins system.

\section{Introduction}

Nonlinear system identification is an important problem in recent years because many interested signals are generated by nonlinear sources or are processed by nonlinear systems. There are several conditions that the inherent nonlinearities and distortions cannot be tolerated, so nonlinear processing techniques need to be employed. Such important examples as nonlinear echo cancellation, predistortion of nonlinear channels, equalization of communication channels, linearization of loudspeaker nonlinearities, enhancement of noisy images, edge extraction, distortions in magnetic recording systems, motion of moored ships in ocean waves, control of industrial processes, physiological models, nuclear fission, and so on (Benedetto \& Biglieri 1983; Frank 1995; Lazzarin et al 1994; Mathews \& Sicuranza 2000; Kalouptsidis et al 2003; Boyd 1985; Kalouptsidis 1997).

The class of nonlinear model is restricted; however polynomial systems form a popular class of nonlinear systems (Mathews \& Sicuranza 2000). This class of systems is identified by inputoutput relationships of the form $\mathrm{Eq}(1)$,

$$
y(n)=f(u(n), u(n-1), \ldots \ldots . . u(n-N), y(n-1), \ldots \ldots \ldots y(n-M)),
$$

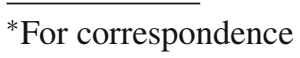


where $u(n)$ and $y(n)$ present the input and output signals, respectively, and $f(\ldots)$ is a polynomial in $N+M+1$ variables. Polynomial systems can be categorized into recursive and non-recursive systems. Non-recursive polynomial systems are acquired that $y(n)$ only depend on $u(n-i), i=0,1, \ldots \ldots, N$. In this case, the system takes the form of a Volterra series (Schetzen 1980). In practical applications Volterra series are properly restricted owing to the large number of coefficients that need to be estimated. Recursive polynomial systems can present many nonlinear systems with greater efficiency and accurately than non-recursive systems. A special class of recursive nonlinear models is the class of bilinear systems. The input-output relationship of a bilinear system is given by $\mathrm{Eq}(2)$,

$$
y(n)=\sum_{i=1}^{K_{a}} a(i) y(n-i)+\sum_{i=0}^{K_{b}} b(i) u(n-i)+\sum_{i=1}^{K_{c y}} \sum_{j=0}^{K_{c u}} c(i, j) y(n-i) u(n-j),
$$

where $a(i), b(i)$ and $c(i, j)$ represent the system coefficients, and the set $\left\{\mathrm{K}_{\mathrm{a}}, \mathrm{K}_{\mathrm{b}}, \mathrm{K}_{\mathrm{cy}}, \mathrm{K}_{\mathrm{cu}}\right\}$ corresponds to the order of the system. As seen Eq (2), the bilinear system is simple and similar nonlinear system to a linear system form. It has many advantages, such as its special variable structure property which makes it advantageous on system optimization and identification. So, several practical systems have been modelled by bilinear systems (Wang \& Gu 2007; Agazzi et al 1982; Bruni et al 1974). Such examples include channel equalization, echo cancellation, nonlinear tracking, electroencephalogram (EEG) signal classification and many other areas of engineering, socioeconomics and biology (Wang \& Gu 2007; Agazzi et al 1982; Bruni et al 1974).

Traditional methods for input-output bilinear system identification are developed as seen in the literature (Mathews \& Sicuranza 2000; Kalouptsidis et al 2003; Koukoulas et al 2002; Mathews \& Moon 1991; Tsoulkas et al 2001). The parameter estimation for bilinear system identification of the methods can be implemented by the Least Mean Square (LMS) algorithm, the extended least squares algorithms, or their variants (Mathews \& Sicuranza 2000). Different approaches using cross-cumulant information and blind identification are applied in (Kalouptsidis et al 2003; Koukoulas et al 2002; Mathews \& Moon 1991). In addition, identification of bilinear systems using global optimization algorithms has attracted limited attention so far (Wang \& Gu 2007; Subudhi \& Jena 2008; Subudhi et al 2008; Karaboga 2005).

Recently, among modern global optimization algorithms, a novel optimization algorithm called Differential Evolution algorithm (DEA) has been proposed (Storn \& Price 1997; Storn 1999; Price \& Storn 1997). This method is presented to optimally solve the optimization problems. DEA is carried out by using real number directly without further binary transformations. Therefore, this method is very convenient to use in most real engineering applications, and is also easily implemented by computer programs. Basically, DEA contains three major operators, first, mutation operator from genetic science, second, crossover and third, selection operator simulating natural selection phenomena to achieve the evolution. By means of these operations, an objective function defined for solving system optimization problem can be minimized. The other modern global optimization algorithm is genetic algorithm (GA). GA is a population-based algorithm like DEA using the similar operators; mutation, crossover and selection. GA can efficiently search large solution spaces due to its parallel structure and the probabilistic transition rules employed in the operators. Because of the features mentioned above, these algorithms have been successfully developed for solving a variety of system optimization problems and applied to a number of different fields (Cheng \& Hwang 2001; Kyprianou et al 2001; Chang 2006; Chang 2007; Price 1999). 
The main contribution of this paper is to propose a novel strategy that is based on using the DEA to parameter estimation of nonlinear bilinear systems. The performance of DEA is also compared with GA. Box-Jenkins system and different type bilinear systems are identified using these algorithms.

The organization of this paper is as follows. Section 2 introduces a brief definition of the problem. Section 3 presents the basic information on DEA and the evolutionary design steps for parameter estimation of bilinear systems. In section 4, some numerical simulations are made to verify the feasibility of the proposed method. Finally, some conclusions are made in section 5 .

\section{Definition of the problem}

Consider the nonlinear bilinear systems with the input-output relationship given by Eq (2). So, the design of these systems can be evaluated as an optimization problem of the cost function $\mathrm{J}(\mathrm{w})$ indicated as follows

$$
\min _{w \in W} J(w)
$$

where $w=\mathrm{a}(\mathrm{i}), \mathrm{b}(\mathrm{i})$ and $\mathrm{c}(\mathrm{i}, \mathrm{j})$ is the bilinear system coefficient vector in Eq (2). The aim of the cost function $J(w)$ is minimized by adjusting $w$. The cost function, called Mean Square Error (MSE), is usually expressed as the time averaged of function defined by Eq (3):

$$
J(w)=\frac{1}{N} \sum_{n=1}^{N}(d(n)-y(n))^{2},
$$

where $d(n)$ and $y(n)$ are the desired and actual responses of the systems, respectively, and $N$ is the number of samples used for the calculation of the cost function. The identification architecture is schematically given in figure 1 for a nonlinear system by using the algorithm.

\section{Differential evolution algorithm}

The DEA has been demonstrated to be an effective method for solving certain optimization problems in literature (Storn 1999; Price \& Storn 1997; Cheng \& Hwang 2001; Kyprianou et al

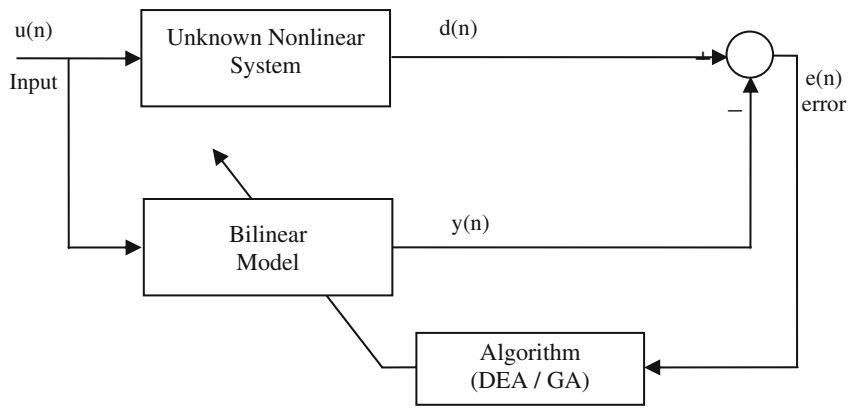

Figure 1. Identification architectures for nonlinear systems by using the algorithm. 
2001; Chang 2006; Chang 2007; Price 1999). Basically, there are three important factors in the use of DEA: the population size NP, the scaling factor $\mathrm{F}$, and the crossover rate $\mathrm{C}$. The main steps of basic DEA are given below.

\begin{tabular}{|l|}
\hline Initialization \\
Evaluation \\
REPEAT \\
Mutation \\
Recombination \\
Evaluation \\
Selection \\
UNTIL (termination criteria are met)
\end{tabular}

In applying the DEA to solve the identification problem for nonlinear bilinear system, firstly, it begins to generate a population of NP parameter vectors whose initial values are randomly generated from the user-defined search region. Then, parameter vectors in the population are exposed to natural selection. In every generation, each population vector becomes a target vector. Each target vector crosses with a donor vector, which is produced by mutating a randomly selected population vector with the difference between two randomly selected population vectors, in order to obtain a trial vector. If the cost function generated by the trial vector is less than that of the target, the target is replaced by the trial vector in the next generation. DEA have three important operators, mutation, crossover, and selection. Mutation, operator is the beginning operation. The mutation operation is defined more clearly that a population vector is mutated into a donor vector by adding the weighted difference of two randomly selected but different population vectors, The generated vector is known as a donor vector to obtain a trial population vector by using crossover operation. Crossover operation produce trial vector. An optimization task consisting of $w$ parameters can be presented. We first give a set of random numbers which are distributed uniformly in the interval $(0,1)$, and another set of three binary sequence is derived. Then each element of a trial vector $\mathrm{T}$ is taken according to the following crossover. Selection operation is the last operation of DEA. In this operation, performances of trial vector and target vector are computed and compared with each other. Then the better one is selected. A generation is complete of mutation, crossover and selection operations. Readers interested in details of DEA may access to an important source on this subject (Storn 1999; Price \& Storn 1997; Storn 1999; Cheng \& Hwang 2001; Kyprianou et al 2001; Chang 2006, 2007; Price 1999).

\section{Simulation results}

In this section, firstly, different types of bilinear systems (Kalouptsidis et al 2003; Zorlu \& Ozer 2004) have been identified using DEA. Secondly, the algorithms have been applied to identification of Box-Jenkins system (Box \& Jenkins 1970) via a bilinear model. To compare the effectiveness of this approach, GA has also been used on the simulations. 


\subsection{Example 1}

In the first example, the unknown system (Zorlu \& Ozer 2004) and the proposed model are as follows:

$$
\begin{aligned}
y(n)= & 0.325 y(n-1)-0.4 u(n-1)+0.56 u(n-3)-u(n-4)-0.65 y(n-2) u(n-2) \\
& +0.065 y(n-3) u(n-5)+0.065 y(n-5) u(n-2), \\
y_{m}(n)= & a_{1} y(n-1)+b_{1} u(n-1)+b_{3} u(n-3)+b_{4} u(n-4)+c_{2,2} y(n-2) u(n-2) \\
& +c_{3,5} y(n-3) u(n-5)+c_{5,2} y(n-5) u(n-2) .
\end{aligned}
$$

In the structures of the system and model; a white Gaussian sequence was used for input, $u(n)$. The bilinear model order is equal to the unknown system order as seen Eqs (4 and 5). In the simulations, 100 data points have been generated by the system.

The effect of the control parameters on the DEA's performance has been studied by Price (1999). The parameter values used in this study have been selected according to the recommended values in that work. Also, in order to carry out the comparison of the algorithms in similar conditions, the values of control parameters of the algorithms have been chosen to be equal to each other. The control parameter values of the algorithms for all examples used in this work are presented. These parameters have been obtained from many trials.

The results obtained from simulations in noiseless case have been shown in table 1 . In this table, the estimation parameter values obtained from the DEA and GA have been also illustrated for accurate comparison. The cost function value (MSE) obtained for DEA and GA are $1.5 \times 10^{-5}$ and $5.6 \times 10^{-5}$, respectively. The variation of MSE-generation has also been presented in figure 2 as graphically for 350 generation number. It is clearly seen from figure 2 that DEA is faster than GA.

\subsection{Example 2}

In the second example, the unknown system (Kalouptsidis et al 2003) and the proposed model are as follows:

$$
\begin{aligned}
y(n)= & -0.1 y(n-1)+0.02 y(n-2)+1 u(n)-0.4 u(n-1)+0.1 y(n-1) u(n-1) \\
& -0.1 y(n-4) u(n-1)-0.05 y(n-2) u(n-2)+0.05 y(n-4) u(n-2) \\
& +0.3 y(n-3) u(n-3)+0.1 y(n-4) u(n-3) . \\
y_{m}(n)= & a_{1} y(n-1)+a_{2} y(n-2)+b_{0} u(n)+b_{1} u(n-1)+c_{1,1} y(n-1) u(n-1) \\
& +c_{4,1} y(n-4) u(n-1)+c_{2,2} y(n-2) u(n-2)+c_{4,2} y(n-4) u(n-2) \\
& +c_{3,3} y(n-3) u(n-3)+c_{4,3} y(n-4) u(n-3) .
\end{aligned}
$$

Table 1. Comparison of the DEA and GA for bilinear model in Eq (5).

\begin{tabular}{lcccccccc}
\hline Parameters & $\mathrm{a}_{1}$ & $\mathrm{~b}_{1}$ & $\mathrm{~b}_{3}$ & $\mathrm{~B}_{4}$ & $\mathrm{c}_{2,2}$ & \multicolumn{1}{c}{$\mathrm{c}_{3,5}$} & $\mathrm{c}_{5,2}$ & \multicolumn{1}{c}{ MSE } \\
\hline True values & 0.325 & -0.400 & 0.560 & -1.000 & -0.650 & 0.065 & 0.065 & - \\
DEA & 0.325 & -0.401 & 0.559 & -0.997 & -0.656 & -0.016 & 0.060 & $1.5 \times 10^{-5}$ \\
GA & 0.326 & -0.394 & 0.567 & -1.009 & -0.612 & 0.003 & 0.044 & $5.6 \times 10^{-5}$ \\
\hline
\end{tabular}




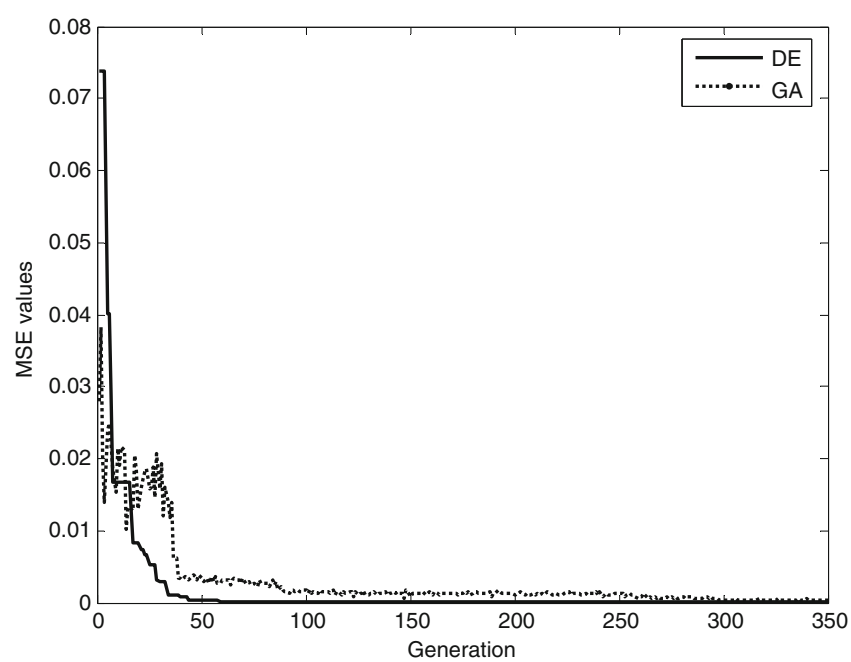

Figure 2. Evolution of the MSE values of the first example for DEA and GA.

In the structures of the system and model; a white Gaussian sequence was used for input, $u(n)$. The bilinear model order is equal to the unknown system order as seen in Eqs (6 and 7). In the simulations, 100 data points have been generated by the system.

The control parameter values used for the algorithms for example 2 are given. These parameters have been obtained from many trials. The results achieved from simulations in the noiseless case have been presented in table 2 . In this table, the estimation parameter values obtained from the DEA and GA have been also illustrated for accurate comparison. The cost function value (MSE) results for the DEA and GA are $7.66 \times 10^{-31}$ and $2.12 \times 10^{-4}$, respectively. In addition, the MSE results have been shown in figure 3 as graphically for 350 generations.

The above results indicate that DEA and GA are suitable for use in bilinear system identification problems. Also, DEA produced better results than GA that can be estimated system parameters with low error. In addition, the results obtained from these algorithms have been found after 20 trails for examples.

Table 2. Comparison of the DEA and GA for bilinear model in Eq (7).

\begin{tabular}{lccc}
\hline Parameters & True values & DEA & GA \\
\hline $\mathrm{a}_{1}$ & -0.1 & -0.1 & 0.13 \\
$\mathrm{a}_{2}$ & 0.02 & 0.02 & 0.11 \\
$\mathrm{~b}_{0}$ & 1.00 & 1.00 & 1.01 \\
$\mathrm{~b}_{1}$ & -0.40 & -0.40 & -0.63 \\
$\mathrm{c}_{1,1}$ & 0.10 & 0.10 & 0.08 \\
$\mathrm{c}_{4,1}$ & -0.10 & -0.10 & -0.06 \\
$\mathrm{c}_{2,2}$ & -0.05 & -0.05 & -0.07 \\
$\mathrm{c}_{4,2}$ & 0.05 & 0.05 & 0.05 \\
$\mathrm{c}_{3,3}$ & 0.30 & 0.30 & 0.28 \\
$\mathrm{c}_{4,3}$ & 0.10 & 0.10 & 0.08 \\
\hline MSE & - & $7.66 \times 10^{-31}$ & $2.12 \times 10^{-4}$ \\
\hline
\end{tabular}




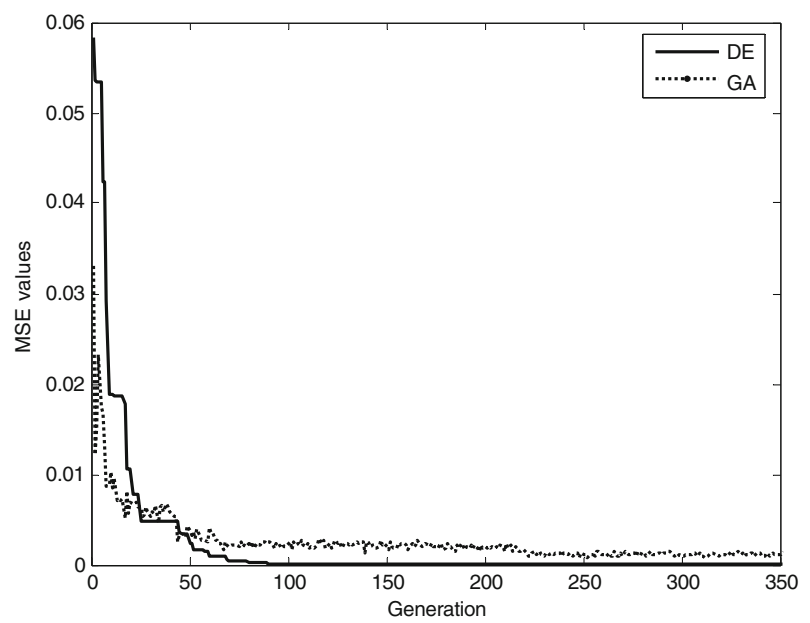

Figure 3. Evolution of the MSE values of the second example for DEA and GA.

\subsection{Example 3}

4.3(a) Noiseless cases: The gas furnace data (series J) of Box-Jenkins (1970) was recorded from a combustion process of a methane-air mixture. It is well-known and frequently used as a benchmark example for testing identification and prediction algorithms. The data set consists of 296 pairs of input-output measurements. The input $u(t)$ is the gas flow into the furnace and the output $y(t)$ is the $\mathrm{CO}_{2}$ concentration in outlet gas. The sampling interval is $9 \mathrm{~s}$. The gas furnace data are available on the web site of the Working Group on Data Modelling Benchmark IEEE Neural Network Council (http://neural.cs.nthu.edu.tw/jang/benchmark/). In literature, many works concerned with Box-Jenkins system are presented (Xu \& Lu 1987; Lee et al 1994; Lin \& Cunningham 1995; Nie 1995; Jang et al 1997; Farag et al 1998). In this work, new and simple parametric nonlinear model is presented for Box-Jenkins system. Parameters of the proposed bilinear model are estimated with DEA, called PMDE, and GA, called PMGA. The proposed model is in the following form

$$
\begin{aligned}
y_{m}(n)= & a_{1} y(n-1)+a_{2} y(n-2)+b_{4} u(n-4)+c_{1,4} y(n-1) u(n-4) \\
& +c_{2,1} y(n-2) u(n-1) .
\end{aligned}
$$

The input, $u(n)$, of the model is the gas flow into the furnace and the output, $y(n)$, is the $\mathrm{CO}_{2}$ concentration. In simulations, 290 data points have been generated by the Box-Jenkins system.

The most important part of the system identification is the process of obtaining the suitable model. If this model is linear model, there are certain criteria in the literature, such as Akaike

Table 3. Estimated parameter values with DEA and GA for bilinear model in Eq (8).

\begin{tabular}{lcccccc}
\hline Model parameters & $\mathrm{a}_{1}$ & $\mathrm{a}_{2}$ & $\mathrm{~b}_{4}$ & $\mathrm{c}_{1,4}$ & $\mathrm{c}_{2,1}$ & $\mathrm{MSE}$ \\
\hline DEA & 1.8524 & -0.8525 & 0.1042 & 0.0027 & -0.0053 & 0.1247 \\
GA & 2.2323 & -1.2398 & 4.8002 & -0.0829 & -0.0039 & 0.3508 \\
\hline
\end{tabular}


Table 4. Comparison of identification errors using different works for the Box-Jenkins system.

\begin{tabular}{lc}
\hline Works reference & MSE \\
\hline Xu's model (Xu \& Lu 1987) & 0.3280 \\
Lee's model (Lee et al 1994) & 0.4070 \\
Lin's model (Lin \& Cunningham 1995) & 0.2610 \\
Nie's model (Nie 1995) & 0.1690 \\
ANFIS model (Jang et al 1997) & 0.1495 \\
Farag's model (Farag et al 1998) & 0.1110 \\
PMDE (Proposed) & 0.1247 \\
PMGA (Proposed) & 0.3508 \\
\hline
\end{tabular}

information criteria (Akaike 1974), but the certain criteria for nonlinear bilinear models have not been offered yet. So, the one proposed a non-linear bilinear model will work through trial and error to reach the best model. The model proposed by authors was considered the criteria listed in the following; firstly, input and output sequences $[y(n-1) y(n-2) u(n-4)]$ of the proposed bilinear model in Eq (8) have been selected as reference studies in the literature $(\mathrm{Xu}$ \& Lu 1987; Lee et al 1994; Lin \& Cunningham 1995; Nie 1995; Jang et al 1997; Farag et al 1998). Secondly, model parameters were a minimum order depending on the values of this delay sequences. Using these criteria, the best bilinear model of Box-Jenkins system was obtained by many trials. These parameters have been obtained from many trials. The results obtained from simulations have been given in tables 3 and 4 . In table 3, the estimation parameter values obtained from the DEA and GA have been also illustrated. In table 4, the MSE results have been compared with different works concerned with the Box-Jenkins system identification (Xu \& Lu 1987; Lee et al 1994; Lin \& Cunningham 1995; Nie 1995; Jang et al 1997; Farag et al 1998). Table 5 shows the control parameter values of the algorithms for example 3. In addition, the outputs of the bilinear models identified by DEA and GA and the actual time-series are shown in figures 4 and 5 , respectively.

In this work, the proposed bilinear model for Box-Jenkins system has been optimized with DEA and GA. As seen from table 3, different parameter values for proposed model have been estimated for the algorithms. As seen in table 4, DEA produced better results than GA in terms of identification error. The results indicate that the proposed model (PMDE) show a good performance in comparison with different works for Box-Jenkins system in the literature. In addition, the results obtained from these algorithms have been found after 20 trails for examples.

Table 5. Control parameters on the algorithms for all examples.

\begin{tabular}{|c|c|c|c|c|c|c|}
\hline \multirow[t]{2}{*}{ Control parameters } & \multicolumn{2}{|c|}{ Example 1} & \multicolumn{2}{|c|}{ Example 2} & \multicolumn{2}{|c|}{ Example 3} \\
\hline & $\mathrm{DE}$ & GA & $\mathrm{DE}$ & GA & $\mathrm{DE}$ & GA \\
\hline Population size & 70 & 70 & 70 & 70 & 50 & 50 \\
\hline Crossover rate & 0.8 & 0.8 & 0.8 & 0.8 & 0.8 & 0.8 \\
\hline Scaling factor $(\mathrm{F})$ & 0.8 & - & 0.8 & - & 0.8 & - \\
\hline Combination factor $(\mathrm{K})$ & 0.8 & - & 0.8 & - & 0.8 & - \\
\hline Mutation rate & - & 0.2 & - & 0.2 & - & 0.2 \\
\hline Upper and low bounds & {$[-2,+2]$} & {$[-2,+2]$} & {$[-2,+2]$} & {$[-2,+2]$} & {$[-10,+10]$} & {$[-10,+10]$} \\
\hline Generation number & 350 & 1000 & 350 & 2000 & 150 & 1000 \\
\hline
\end{tabular}




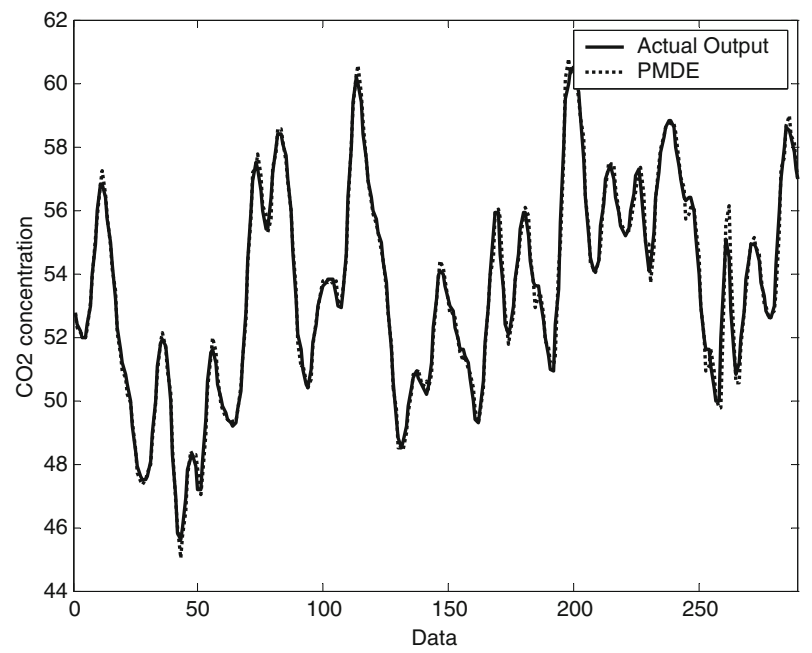

Figure 4. Output of the bilinear model identified with DEA and the actual time-series.

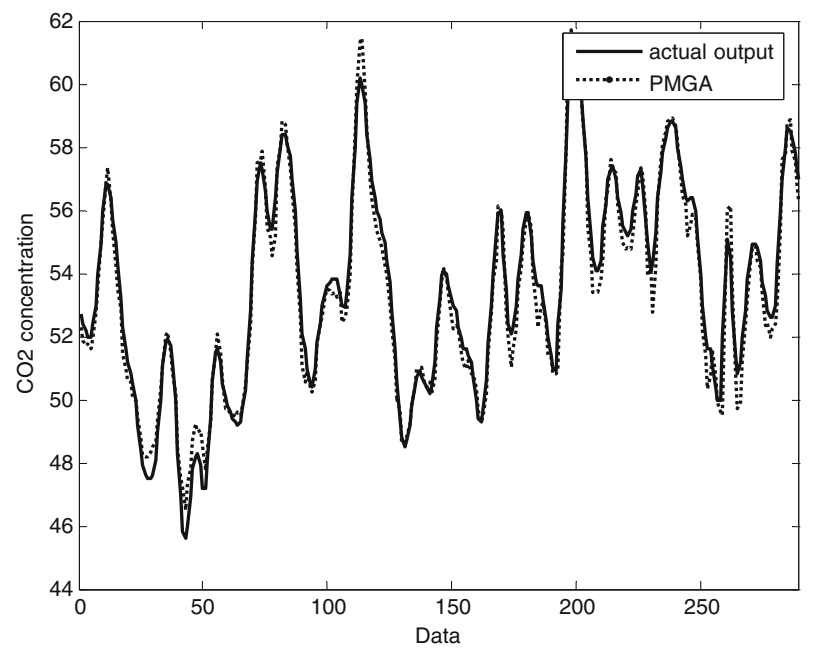

Figure 5. Output of the bilinear model identified with GA and the actual time-series.

Table 6. Estimated parameter values with DEA and GA for bilinear model in Eq (9).

\begin{tabular}{lcccccr}
\hline Model parameters & $\mathrm{a}_{1}$ & $\mathrm{a}_{2}$ & $\mathrm{~b}_{4}$ & $\mathrm{c}_{1,4}$ & $\mathrm{c}_{2,1}$ & MSE \\
\hline DEA & 1.2093 & -0.2102 & 0.4099 & -0.0069 & -0.0058 & 0.2975 \\
GA & 0.8064 & 0.1917 & 1.2429 & -0.0253 & -0.0056 & 0.5872 \\
\hline
\end{tabular}




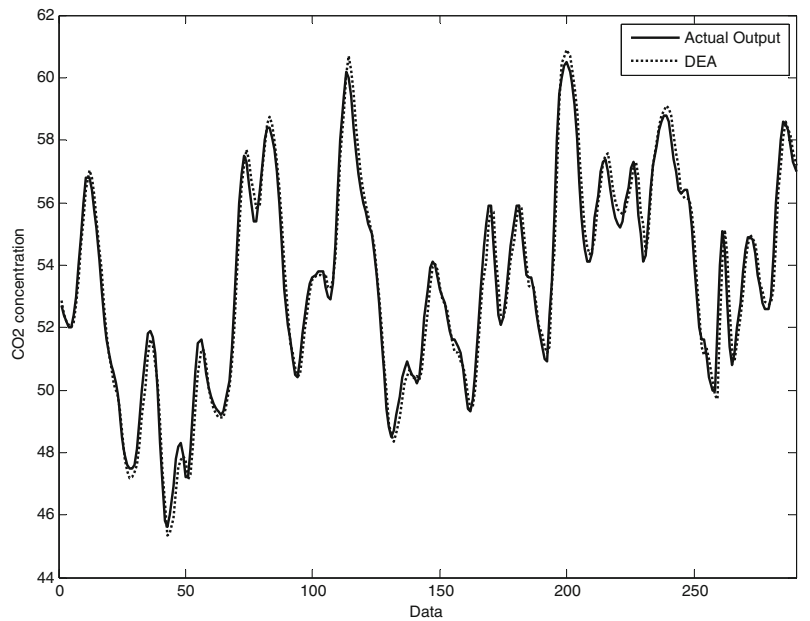

Figure 6. Output of the bilinear model identified with DEA and the actual time-series noisy environments.

4.3(b) Noisy cases: In this example, performance of the proposed bilinear model for BoxJenkins system was carried out in noisy environments. Parameters of the proposed bilinear model are estimated with DEA and GA. The proposed model is of the following form

$$
\begin{aligned}
y_{m}(n)= & a_{1} y(n-1)+a_{2} y(n-2)+b_{4} u(n-4)+c_{1,4} y(n-1) u(n-4) \\
& +c_{2,1} y(n-2) u(n-1)+g(n) .
\end{aligned}
$$

The input, $u(n)$, of the model is the gas flow into the furnace, the output, $\mathrm{y}(\mathrm{n})$, is the $\mathrm{CO}_{2}$ concentration and the noise, $g(n)$, is zero-mean, unit-variance, $40 \mathrm{~dB}$ SNR (Signal-Noise Ratio) Gaussian noise. In simulations, 290 data points have been generated by the Box-Jenkins system.

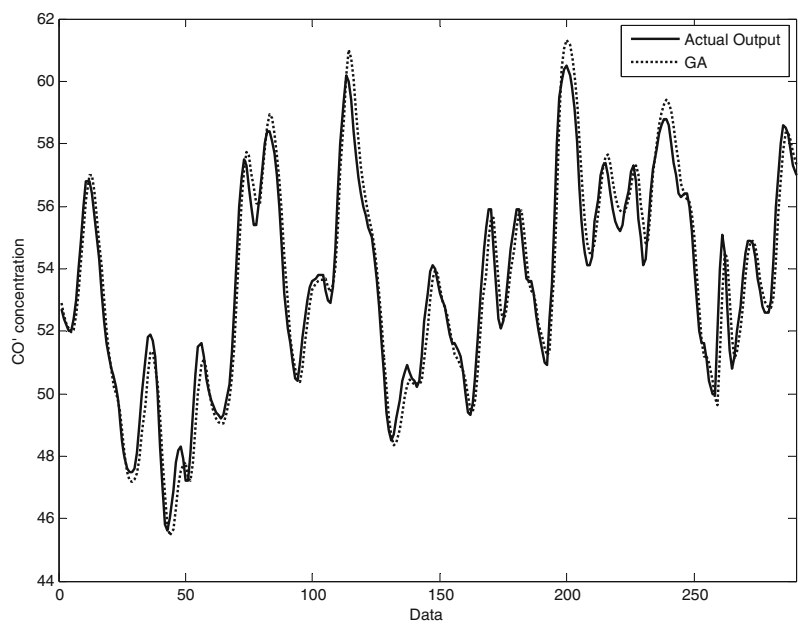

Figure 7. Output of the bilinear model identified with GA and the actual time-series noisy environments. 
This model has been obtained from many trials. The control parameter values of the algorithms are same as the noiseless case (example 4). The results obtained from simulations in noisy environments have been given in table 6 . In the table, the estimation parameter values obtained from the DEA and GA have been also illustrated. In addition, the outputs of the bilinear models identified by DEA and GA and the actual time-series are shown in figures 6 and 7, respectively.

In this work, the proposed bilinear model for Box-Jenkins system has been optimized in nosy situation with DEA and GA. The results demonstrated that DEA show better performance than GA in noisy environments. In addition, the results obtained from these algorithms have been found after 20 trails for examples.

\section{Conclusions}

This study presents a comprehensive comparison of bilinear system identification using differential evolution and genetic algorithms. The performances of the algorithms have been evaluated by computer simulations. In the simulations, Box-Jenkins system and different type bilinear systems have been identified using these algorithms. From the simulation results, bilinear systems can be successfully identified using both the algorithms.

In this paper, the performance comparison of DEA and GA has been realized as well. As a result of this performance comparison, DEA has produced better results than GA. Although GA is the best known evolutionary algorithm which can be easily applicable to these types of the problems, DEA also seems to be successful in the application of the identification problem providing fast convergence. Therefore, DEA seems to be a promising approach for this type of optimization problems.

\section{References}

Agazzi O, Messerschmitt D, Hodges D 1982 Nonlinear echo cancellation of data signals, IEEE Trans. Commun. COMM-30: 2421-2433

Akaike H 1974 A new look at the statistical model identification, IEEE Transactions on Automatic Control 19(6): 716

Benedetto S, Biglieri E 1983 Nonlinear equalization of digital satellite channels, IEEE J. Select. Areas Commun. SAC-1: 57

Box G E P, Jenkins G M 1970 Time series analysis, forcasting and control. (San Francisco, CA: Holden Day)

Boyd S 1985 Volterra series: Engineering fundamentals, Ph.D. dissertation, Univ. California, Berkeley

Bruni C, DiPillo G, Koch G 1974 Bilinear systems: an appealing class of 'nearly linear systems' in theory and applications, IEEE Trans. Automat. Contr. AC-19: 334-348

Chang W D 2006 Parameter identification of Rossler's chaotic system by an evolutionary algorithm, Chaos, Solutions and Fractals 29: 1047-1053

Chang W D 2007 Parameter identification of Chen and Lü systems: A differential evolution approach, Chaos, Solutions and Fractals 32: 1469-1476

Cheng S L, Hwang C 2001 Optimal approximation of linear systems by a differential algorithm, IEEE Trans Syst Man Cybernet-Part A Syst Humans 698-707

Farag W A, Quintana V H, Lambert-Torres G 1998 A genetic-based neuro-fuzzy approach for modeling and control of dynamical systems, IEEE Transactions On Neural Networks. 9: 756-767

Frank W A 1995 An efficient approximation to the quadratic Volterra filter and its application in real-time loudspeaker linearization, Signal Process. 45: 97-114

Jang J S R, Sun C T, Mizutani E 1997 Neuro-fuzzy and soft computing: a computational approach to learning and machine intelligence, (Upper Saddle River, NJ: Prentice-Hall) 
Kalouptsidis N 1997 Signal processing systems: Theory and design (New York: Wiley)

Kalouptsidis N, Koukoulas P, Mathews V J 2003 Blind identification of bilinear systems, IEEE Transactions On Signal Processing 51: 484-499

Karaboga N 2005 Digital IIR Filter Design Using Differential Evolution Algorithm, EURASIP Journal on Applied Signal Processing 8: 1269-1276

Koukoulas P, Tsoulkas V, Kalouptsidis N 2002 A cumulant based algorithm for the identification of input output quadratic systems, Automatica 38: 391-407

Kyprianou A, Worden K, Panet M 2001 Identification of hysteretic systems using the differential evolution algorithm, J. Sound Vib. 289-314

Lazzarin G, Pupolin S, Sarti A 1994 Nonlinearity compensation in digital radio systems, IEEE Trans. Commun. 42: 988-999

Lee Y C, Hwang E, Shih Y P 1994 A combined approach to fuzzy model identification, IEEE Transactions on Systems, Man and Cybernetics 24: 736-744

Lin Y, Cunningham G A 1995 A new approach to fuzzy-neural system modeling, IEEE Transactions on Fuzzy Systems 3: 190-197

Mathews V J, Moon T K 1991 Parameter estimation for a bilinear time series model, in Proc. IEEE Int. Conf. Acoust. Speech Signal Process. 3513-3516

Mathews V J, Sicuranza G L 2000 Polynomial signal processing. (New York: Wiley)

Nie J 1995 Constructing fuzzy model by self-organising counter propagation network, IEEE Transactions on Systems Man and Cybernetics 25: 963-970

Price K V 1999 An introduction to differential evolution, in D Corne, M Dorigo, and F Glover, (eds), New Ideas in Optimization, chapter 6, London, UK: McGraw Hill

Price K, Storn R 1997 Differential evolution, Dr Dobb's Journal 18-24

Schetzen M 1980 The volterra and wiener theories of nonlinear systems. (New York: Wiley)

Storn R 1999 System design by constraint adaptation and differential evolution, IEEE Trans. Evolutn. Comput. 3: 5-9

Storn R, Price K 1997 Differential evolution-a simple and efficient heuristic for global optimization over continuous spaces J. Global Optim. 11: 341-59

Subudhi B, Jena D 2008 Differential Evolution and Levenberg Marquardt Trained Neural Network Scheme for Nonlinear System Identification, Neural Process Lett. 285-296

Subudhi B, Jena D, Gupta M M 2008 Memetic Differential Evolution Trained Neural Networks for Nonlinear System Identification, IEEE Region 10 Colloquium and the Third International Conference on Industrial and Information Systems, Kharagpur, INDIA, 8-10

Tsoulkas V, Koukoulas P, Kalouptsidis N 2001 Identification of input output bilinear systems using cumulants, IEEE Trans. Signal Processing 49: 2753-2761

Wang Z, Gu H 2007 Parameter Identification of Bilinear System Based on Genetic Algorithm, LNCS 4688 83-91

Xu C W, Lu Y Z 1987 Fuzzy model identification and self-learning for dynamic systems, IEEE Transactions on Systems, Man and Cybernetics 17: 683-689

Zorlu H, Ozer S 2004 Yapay Sinir Ağları ile Bilinear Sistemlerin Kimliklendirilmesi (Bilinear System Identification with Artificial Neural Network), ASYU-INISTA, Turkey 179-182 